\title{
Contributions of myocardial ischemia and heart rate to ST segment changes in patients with or without coronary artery disease
}

\author{
S. Häggmark ${ }^{1}$, M. F. Haney ${ }^{2}$, G. Johansson ${ }^{2}$, S. Reiz ${ }^{3}$ and U. Näslund ${ }^{4}$ \\ ${ }^{1}$ Heart Centre, ${ }^{2}$ Anesthesiology and Intensive Care Medicine, University Hospital, Umeå, Sweden, ${ }^{3}$ Anesthesiology, Centre Anti-Douleurs, \\ Clinique de Montchoisi, Lausanne, Switzerland and ${ }^{4}$ Cardiology, Heart Centre, University Hospital, Umeå, Sweden
}

Background: ST changes related to ischemia at different heart rates (HRs) have not been well described. We aimed to analyze ST dynamic changes by vectorcardiography (VCG) during pacing-induced HR changes for subjects with proven coronary artery disease (CAD) and without (non-CAD).

Methods: Symptomatic CAD patients scheduled for elective surgery were enrolled along with a non-CAD group. During anesthesia, both groups were placed at multiple ascending levels. VCG ST data, and in particular in ST change vector magnitude (STC-VM) from baseline, along with arterial and great coronary artery vein (GCV) blood samples were collected to determine regional myocardial lactate production.

Results: A total of $35 \mathrm{CAD}$ and 10 non-CAD patients were studied over six incremental 10 beat/min HR increases. STC-VM mean levels increased in the CAD group from $9 \pm 5$ to $131 \pm 37 \mu \mathrm{V}$ (standard deviation) compared with non-CAD subjects with $8 \pm 3-76 \pm 34 \mu \mathrm{V}$. Myocardial ischemia (lactate production) was noted at higher HRs and the positive predictive value for STC-VM to detect ischemia was $58 \%$ with the negative predictive value being $88 \%$. STC-VM at $54 \mu \mathrm{V}$ showed a sensitivity of $88 \%$ and a specificity of $75 \%$ for identification of ischemia.

Conclusions: Both HR and ischemia at higher HRs contribute to VCG ST elevation. Established ST ischemia detection concerning HR levels is suboptimal, and further attention to the effects of HR on ST segments is needed to improve electrocardiographic ischemia criteria.

Accepted for publication 29 August 2007

Key words: Myocardial ischemia; ischemic heart disease; electrocardiography; vectorcardiography.

(C) 2007 The Authors

Journal compilation (C) 2007 The Acta Anaesthesiologica Scandinavica Foundation
E LECTROCARDIOGRAPHY (ECG) is widely used as a non-invasive method to detect and monitor Myocardial ischemia $(1,2)$. Continuous vectorcardiography (VCG) is one form of ECG that allows measurement of both ST segment magnitude and vector direction change (3). ECG ST analysis, similar to all diagnostic methods, has limitations concerning sensitivity and specificity for ischemia.

This study concerns the detection of ischemia and the relation of electrocardiographic ST changes to heart rate (HR) both during ischemia and in nonischemic conditions. ST changes in relation to increased HR have been reported, even in patients who do not have coronary artery disease (CAD) (4),

Department to which the work should be attributed: Anesthesiology and Intensive Care Medicine, University Hospital of Umea, Umea, Sweden though the mechanism for this is unclear. Different methods for improvement of the diagnostic accuracy of ST analysis, including adjustments for $\mathrm{HR}$, have been proposed (5-9) generally in the context of exercise testing for ischemia detection, when a reference method for detection of ischemia is not present. ST segment deviation can occur independently, or in the absence of increased HRs, although it is common that when myocardial ischemia occurs, HR increases as well. In the clinical context of ischemia diagnosis, interpretation of ST changes, where ischemia is strongly suspected based on risk factors or other indicators, is made more difficult by the presence of high HRs. The specificity of VCG ST changes for ischemia at different HRs has not been well established, and a ST change in magnitude or threshold for ischemia has not been well validated. 


\section{S. Häggmark et al.}

We hypothesized that changes in VCG ST vector parameters during increased $\mathrm{HR}$ can be distinguished from changes due to myocardial ischemia, and that the relative ST change vector magnitude (STC-VM) can detect progress of myocardial ischemia at higher HRs. We aimed to measure and compare ST vector changes during pacing-induced HR changes in subjects with and without myocardial ischemia.

\section{Materials and methods}

\section{Inclusion and exclusion criteria}

With approval from the Umeå University Research Ethics Committee, 54 pre-operative adult thoracic or vascular surgery patients, who met the inclusion criteria and provided verbal informed consent, were included in the study either in a group for symptomatic CAD scheduled for elective coronary bypass surgery $(n=44)$ or in a group without CAD $(n=10)$. Inclusion criteria for the CAD group included disabling angina pectoris, with a positive exercise ECG and coronary angiogram with 'two or three vessel' disease, including coronary artery stenosis in the left anterior descending (LAD) artery, no pathological $Q$ waves pre-operatively and no left ventricular hypertrophy as defined by ECG or by echocardiography. Subjects from the CAD group were excluded from further study if they had signs of myocardial ischemia before or at the start of the protocol, non-sinus rhythm, left- or right-bundle branch block, or if they did not complete at least three pacing steps. Inclusion criteria for the non-CAD group included scheduled elective cardiac valve replacement or abdominal aortic surgery without signs of CAD (no angina), with normal coronary angiogram and no pathological $\mathrm{Q}$ waves pre-operatively (normal rest ECG). Of the 44 CAD subjects included in the study, four subjects were excluded due to pacing non-capture before completing a third pacing step. Another five CAD subjects were excluded because non-ischemia conditions at the baseline pacing measurement could not be confirmed (by both ECG and lactate measurements).

\section{Preparation}

All subjects received a standard premedication (morphine and scopolomine), and pre-operative $\beta$-receptor blocking drugs (10) and nitroglycerin orally were given as ordered by the primary physician the morning before surgery. The anesthesia was standardised (intravenous, opiate and benzodiazepine based) and managed by the responsible anesthesia personnel. Arterial, central venous and pulmonary artery catheters (all three Arrow, Reading, PA) were placed. Intraoperative normoxia and normocapnia were confirmed using intermittent blood gas analysis (ABL 5, Radiometer, Copenhagen, Denmark). Before the start of surgery, a coronary sinus catheter, which had a bipolar pacing electrode $2 \mathrm{~cm}$ from the tip (CCS-7U-90A, Webster Labs, Altadena, CA), was placed into the great cardiac vein (GCV) using fluoroscopy and contrast (Omnipaque ${ }^{\circledR}$, Nycomed, Oslo, Norway).

\section{Measurements}

Blood pressures were recorded (Siemens Sirecust 1280, Dräger Medical, Lubeck, Germany). Blood flow in the GCV was measured using the retrograde thermodilution technique (11) (CBA-210 Wheatestone bridge, Webster Lab. Inc.) and a multi-channel amplifier/graphic recorder (Graphtec Linearcorder WR 3310, Graphtec Corp. Yokohama, Japan).

A 12-lead ECG (Mingograph 62, Siemens-Elema $A B$, Stockholm, Sweden) was recorded for each measurement sequence. The sum of absolute ST deviation values from all 12 leads $\left(\mathrm{ECGST}_{\text {sum }}\right)$ was measured as well as the separate sum for leads V5 and II, derived from the same 12-lead ECG recordings (12). Less than $0.5 \mathrm{~mm}(0.05 \mathrm{mV}) \mathrm{ST}$ change in a single lead was measured as zero change. We averaged measures of ST change from three heart cycles.

Eight additional electrodes were placed according to the lead system for VCG described by Frank (13) and signals at $500 \mathrm{~Hz}$ were recorded (MIDA 1000, 2.74 software, Ortivus Medical, Täby, Sweden). Heart cycles were averaged for periods of $15 \mathrm{~s}$ in three orthogonal planes (X, Y, and Z), and the VCG ST measurement was the combined magnitude (and direction) of these three orthogonal signals $(1,14)$. Lactate concentration was measured for arterial and coronary sinus whole blood (Yellow Springs Lactate Analyzer 1500, OH) for each pacing step.

\section{Protocol}

Pacing (Pacesetter 3077, Osypka, Rheinfeld-Herten, Germany) was started at anHR that was 1015 beats/min (b.p.m.) higher than the subjects' resting HR. A baseline VCG-averaged complex was established, then a second VCG measurement was acquired at the same HR. After this, $6 \mathrm{~min}$ 
pacing steps with 10 b.p.m. increments were employed. A set of general circulatory measurements was acquired, including a 12-lead ECG and GCV flow at the end of each HR period. Arterial and GCV blood samples were drawn at 5 min into each HR period. Whole-blood lactate analyses were immediately performed in the operating room, to determine whether net lactate production in the coronary circulation was evident. The protocol was ended if myocardial ischemia was confirmed either by [lactate gcv $]>\left[\right.$ lactate $\left._{\text {arterial }}\right]$ or by ST changes $(\geq 0.2 \mathrm{mV})$ in two adjacent leads in the 12-lead ECG (a different ST measurement level was defined for the purposes of analysis of results). If no myocardial ischemia was detected, the pacing level was increased by steps of 10 b.p.m. with measurements until either pacing was characterised by non-capture or lactate production/significant ECG ST changes occurred.

\section{VCG ST measurements}

VCG ST magnitudes were calculated using the following formulae (1): ST-VM $=\sqrt{X^{2}+Y^{2}+Z^{2}}$ in microvolts $(\mu \mathrm{V})$. The ST vector change angle (STC-VA) was calculated as the angle between the reference ST vector and the current ST vector. The relative spatial difference between the reference and the current ST vector was calculated as follows:

$$
\mathrm{STC}-\mathrm{VM}=\sqrt{\left(X_{i}-X_{0}\right)^{2}+\left(Y_{i}-Y_{0}\right)^{2}+\left(Z_{i}-Z_{0}\right)^{2}}
$$

in $\mu \mathrm{V}$ with 0 (baseline reference) and $i$ (current) measurements. The median VCG ST point during the last minute of each pacing step was chosen for measurement. All ST measurements were made at $\mathrm{J}+20 \mathrm{~ms}$. Vector planes and a representative example of orthogonal lead and ST signals are shown in Fig. 1 to illustrate the derivation of the VCG ST measurements.

\section{Analysis and calculations}

Left ventricular myocardial oxygen consumption $\left(\begin{array}{ll}\mathrm{LV} & \mathrm{MVO}_{2}\end{array}\right)$ was calculated as GCV flow $\times$ myocardial arterio-venous oxygen content difference $\left(\mathrm{ml} \mathrm{O} \mathrm{O}_{2} \times \min ^{-1}\right)$ (15). Myocardial lactate extraction (MLE, \%) was calculated as ([lactate $\left.]_{\text {arterial }}-[\text { lactate }]_{\mathrm{GCV}}\right) /$ /lactate $]_{\text {arterial }} \times 100(\%)$. Transcoronary lactate flux was calculated as ([lactate $\left.]_{\text {arterial }}-[\text { lactate }]_{\mathrm{GCV}}\right) \times \mathrm{GCV}$ flow $\left(\mathrm{ml} \times \mathrm{min}^{-1}\right)$. For post hoc analysis of myocardial ischemia by 12-lead ECG, a threshold of ST change $\geq 1 \mathrm{~mm}$ in two adjacent precordial leads was used (16). Rate-pressure product (RPP) was calculated as $\mathrm{HR} \times$ systolic arterial blood pressure.

Myocardial ischemia by lactate analysis was defined as decreasing MLE, which was also at levels less than $10 \%$ (17-19). In subjects where progressive lactate production was documented confirming adequate coronary sinus sampling of ischemic myocardial areas, pacing steps before ischemia (by lactate results) were categorised as non-ischemia.

\section{Statistical analysis}

Measured values are presented as the mean \pm standard deviation (SD). Repeated measures ANOVA were used to analyse relations between serial measures at different pacing steps. Paired $t$-testing was used to test for differences for paired observations, and between-groups testing was used for grouped measurement with Bonferroni correction for multiple comparisons. For comparison of frequencies, Fisher's exact test or the $\chi^{2}$ test was used. A $P$-value of $<0.05$ was used for statistical significance. ROC analysis was performed to assess the relationship between ischemia (by lactate assessment) and VCG ST levels. Positive predictive value was calculated as the number of true positive (TP) observations divided by the sum of all positive observations, both true and false positives. Negative predictive value was calculated as the number of true negative (TN) observations divided by the sum of all negative observations, both true and false negative.

\section{Results}

Thirty-five CAD and 10 non-CAD subjects completed the pacing and measurement protocol for at least three pacing steps, and were included in the analysis. There were no peri-operative myocardial infarctions diagnosed by the clinical teams in any of the subjects as defined by local ECG and enzyme criteria, which were routinely checked in all postoperative patients, and all subjects were alive at 1 year following surgery.

The CAD group was older, had more medical history and medications, and lower functional status than the non-CAD group (Table 1). The non-CAD group included aortic valve replacement $(n=7)$ and abdominal aortic surgery patients $(n=3)$. Five of the 10 subjects in the non-CAD group met criteria for ventricular hypertrophy by 


\section{S. Häggmark et al.}
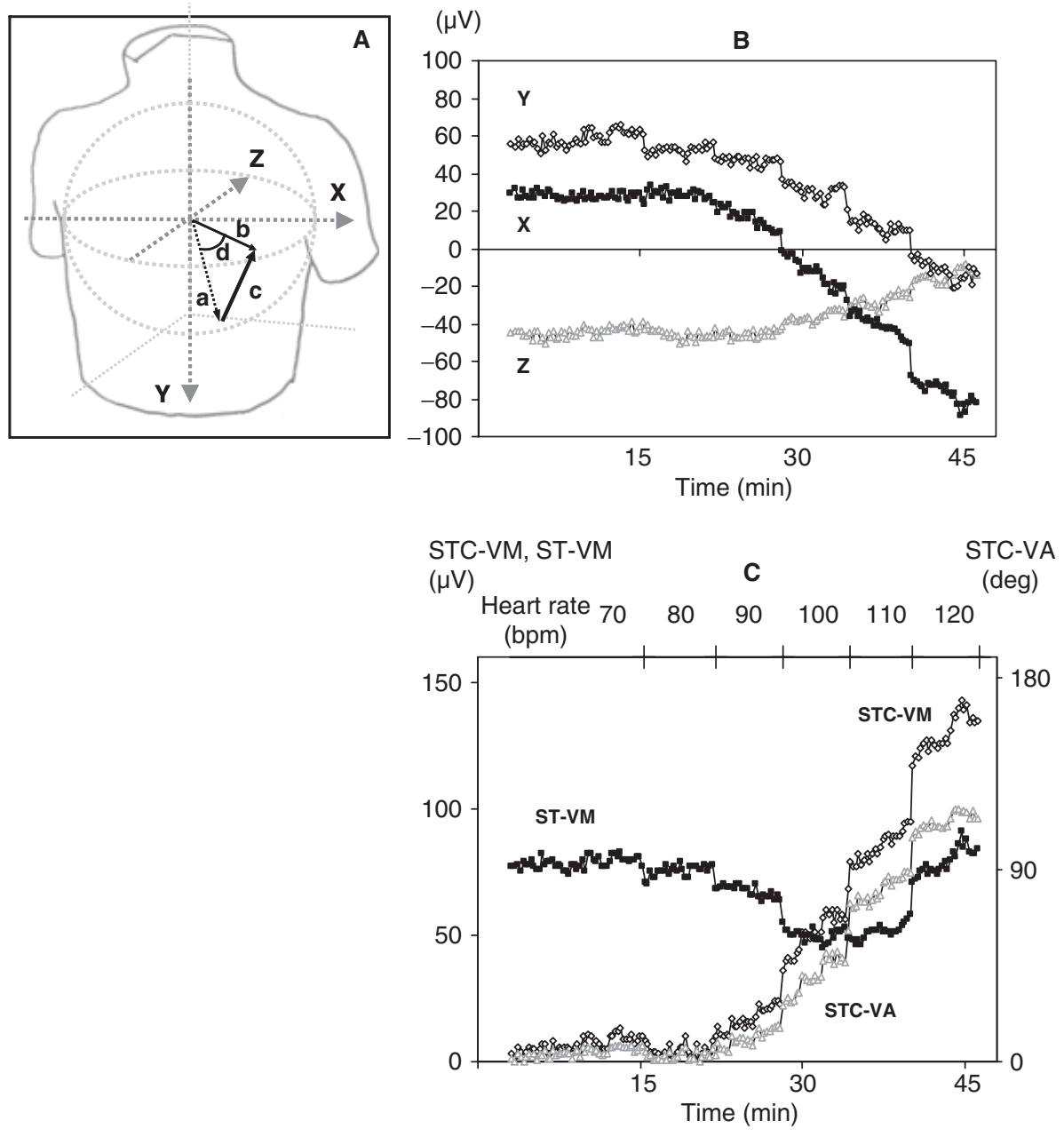

Fig. 1. The ST vector represents the sum of all electrical forces measured $20 \mathrm{~ms}$ after the J-point. (A) (a) represents the first recorded ST vector (the reference), (b) represents the current ST vector. ST vector magnitude (ST-VM) is the length of the current ST vector. STC-VM is the spatial distance between the reference $S T$ vector (a), and the current ST vector (b). STC-VA $(d)$ is the spatial angle between the reference ST-vector (a) and the current ST vector (b). (B) orthogonal leads $(\mathrm{x}, \mathrm{y}$, and $\mathrm{z})$ are shown for $a$ representative pacing sequence in a coronary artery disease subject. These orthogonal leads are used to calculate vector length and angle, which are presented in (C). Ischemia, in this subject, was noted (by post hoc lactate analysis), first at heart rate 100 b.p.m.

pre-operative echocardiography, though not by ECG. The CAD and non-CAD groups differed for HRs at baseline (Table 2) and responded to pacing with changes in $\mathrm{LV} \mathrm{MVO}_{2}$ and GCV flow.

\section{Pacing}

In the CAD group, 13 subjects reached a pacing level where they demonstrated incomplete capture. Thirty-four of 35 CAD subjects demonstrated ischemia either by ECG ST deviation or by lactate production. When all $35 \mathrm{CAD}$ subjects and all pacing levels (excluding baseline) were combined, there were 167 measurement points, and of these 80 were positive for ST deviation in the 12-lead ECG, and 55 were positive for ischemia by lactate criteria. Forty-one positive ischemia events were positive both for lactate and the ECG. From all CAD subjects, 28/35 demonstrated positive lactate production during one or more of the pacing steps. For all measurement sequences (167), there were 17 positive ECG events with no positive lactate, and
14 positive lactate events had no 12-lead ECG ST changes indicative of ischemia.

In the non-CAD group, there were 57 measurements (excluding baseline). Of these, 11/57 measurements (including eight measurements from hypertrophy subjects) and six of 10 subjects at some point in the pacing sequence demonstrated ST change ( $>1 \mathrm{~mm}$ in two adjacent leads) in the 12lead ECG, while one showed myocardial ischemia by lactate criteria.

At max pacing levels, mean 12-lead ECGST sum $_{\text {s. }}$ (all leads) for the non-CAD group $(n=10)$ was $0.07 \mathrm{~mm} \pm 0.04(\mathrm{SD})$, and for the CAD group, it was $1.07 \pm 0.44 \mathrm{mV}$. At the same max pacing levels, the sum of ST change in leads II and V5 was $0.01 \pm 0.01$ for the non-CAD group, and $0.24 \pm 0.10 \mathrm{mV}$ for the CAD group.

\section{Transcoronary lactate during pacing}

For the non-CAD group, there was more transcoronary lactate consumption at higher HRs (Table 2, data shown for baseline and peak pacing levels only), 
while in the CAD group lactate extraction decreased and regional myocardial lactate production (grouped) developed at HR baseline +30 and above. Twenty-six subjects in the CAD group exhibited decreasing and

Table 1

Subject characteristics by group.

\begin{tabular}{|c|c|c|}
\hline & $\begin{array}{l}\text { CAD } \\
\text { group }\end{array}$ & $\begin{array}{l}\text { Non-CAD } \\
\text { group }\end{array}$ \\
\hline & $(n=35)$ & $(n=10)$ \\
\hline Age (years) & $61 \pm 8$ & $52 \pm 4^{a}$ \\
\hline Age, range (years) & $44-73$ & $45-59$ \\
\hline Gender, male $(n)$ & 26 & 9 \\
\hline $\mathrm{BMI}\left(\mathrm{kg} \mathrm{m}^{-2}\right)$ & $26.2 \pm 3.2$ & $25.7 \pm 2.4$ \\
\hline \multicolumn{3}{|l|}{ Previous medical history $(n)$} \\
\hline Angina Pectoris & 35 & $0^{\mathrm{b}}$ \\
\hline Myocardial Infarction & 15 & $0^{\mathrm{b}}$ \\
\hline Hypertension & 16 & 5 \\
\hline Diabetes & 3 & 0 \\
\hline \multicolumn{3}{|l|}{ NYHA function class $(n)^{\mathrm{c}}$} \\
\hline I & 0 & 8 \\
\hline II & 2 & 2 \\
\hline III & 33 & 0 \\
\hline \multicolumn{3}{|l|}{ Cardiac medication $(n)$} \\
\hline$\beta$ adrenergic blocking drugs & 25 & $2^{b}$ \\
\hline Calcium channel blocking drugs & 12 & 1 \\
\hline ACE-inhibitors or All antagonists & 13 & 4 \\
\hline Nitrates & 33 & $0^{\mathrm{b}}$ \\
\hline Diuretics & 1 & 0 \\
\hline Vasodilators & 7 & 0 \\
\hline Digitalis & 0 & 0 \\
\hline Antiarrytmic drugs & 0 & 0 \\
\hline \multicolumn{3}{|c|}{$\begin{array}{l}{ }^{a} P<0.05 \text { with } t \text {-test between groups. } \\
{ }^{\mathrm{b}} P<0.05 \text { with Fishers' exact test. } \\
{ }^{\mathrm{c}} P<0.05 \text { with } \chi^{2} \text { test. } \\
\text { BMI, body mass index; NYHA, New York Heart Association; } \\
\text { ACE, angiotensin converting enzyme; CAD, coronary artery } \\
\text { disease; SD, standard deviation. Data are presented as } \\
\text { mean } \pm \text { SD or number of patients. }\end{array}$} \\
\hline
\end{tabular}

low-lactate extraction, progressing toward and reaching (in 22/26) absolute lactate production.

\section{ST-VM, STC-VA, STC-VM and ECGST sum $_{\text {. }}$}

ST-VM (Fig. 2) for the CAD group showed a broad range of initial values, which increased at highest HRs, although individual ST-VM courses were heterogeneous, and this was not well represented by mean ST-VM values. STC-VM demonstrated increases related to HR steps from baseline at the highest three HRs for CAD subjects compared with non-CAD subjects. STC-VA demonstrated a similar pattern with greater increases for pacing step at the highest two HRs for CAD compared with non-CAD groups.

\section{ST-VM, STC-VM and ischemia}

The relation of ischemia (presence or absence defined by lactate technique) to ST-VM and STC$\mathrm{VM}$ observations (all patients, all sequences) was analyzed using ROC (Fig. 3). The absolute ST-VM level demonstrated a weak relation to ischemia (area under the ROC curve $0.61,95 \%$ CI of area $0.52-0.70, P=0.008)$. The STC-VM level, on the other hand, had a stronger relation to ischemia (area under the ROC curve 0.862, 95\% CI of area $0.81-0.92, P<0.0001)$. The STC-VA-level relation to ischemia was also analyzed using ROC (area under curve $0.83,95 \% \mathrm{CI}$ of area $0.77-0.88, P<0.0001$ ). The STC-VM relation to ischemia was further analyzed for predictive value for ischemia incorporating baseline levels plus $50 \mu \mathrm{V}$ as suggested by other investigators $(20,21)$ as an ischemic limit, as

Table 2

Circulatory and cardiac parameters for baseline and highest pacing level.

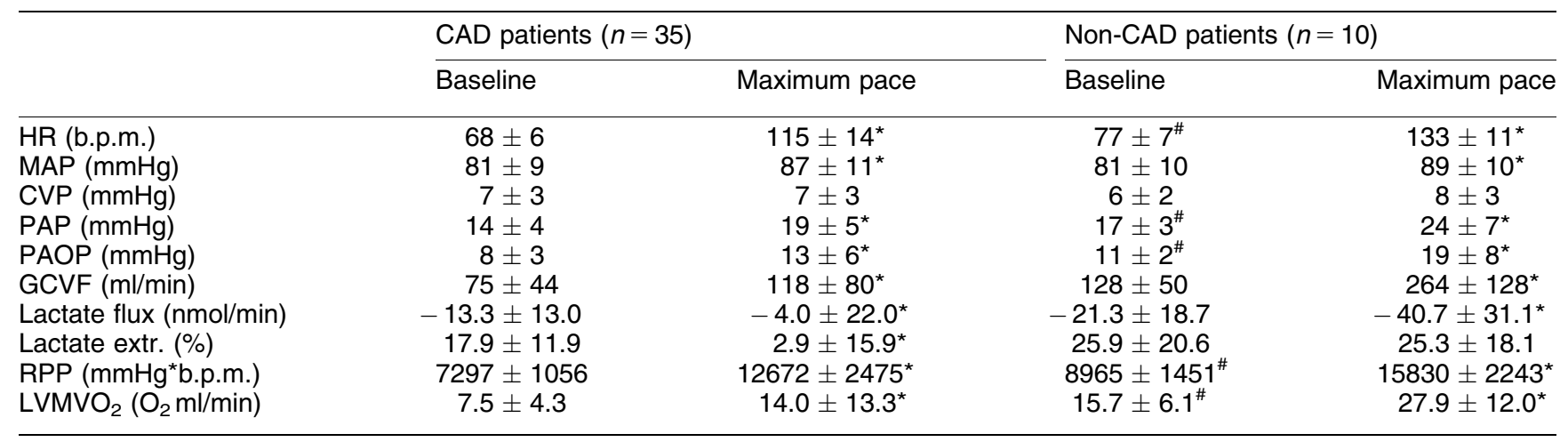

*Denotes significant difference $P<0.05$ between paired measurements baseline and maximum pace.

\#Denotes comparison between groups at baseline.

HR, heart rate; MAP, mean arterial pressure; CVP, central venous pressure; PAP, mean pulmonary artery pressure; PAOP, mean

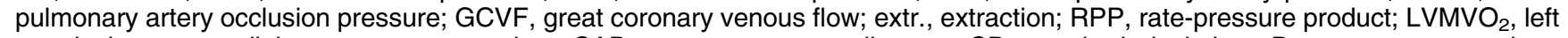
ventricular myocardial oxygen consumption; CAD, coronary artery disease; SD standard deviation. Data are presented as mean \pm SD. 


\section{S. Häggmark et al.}
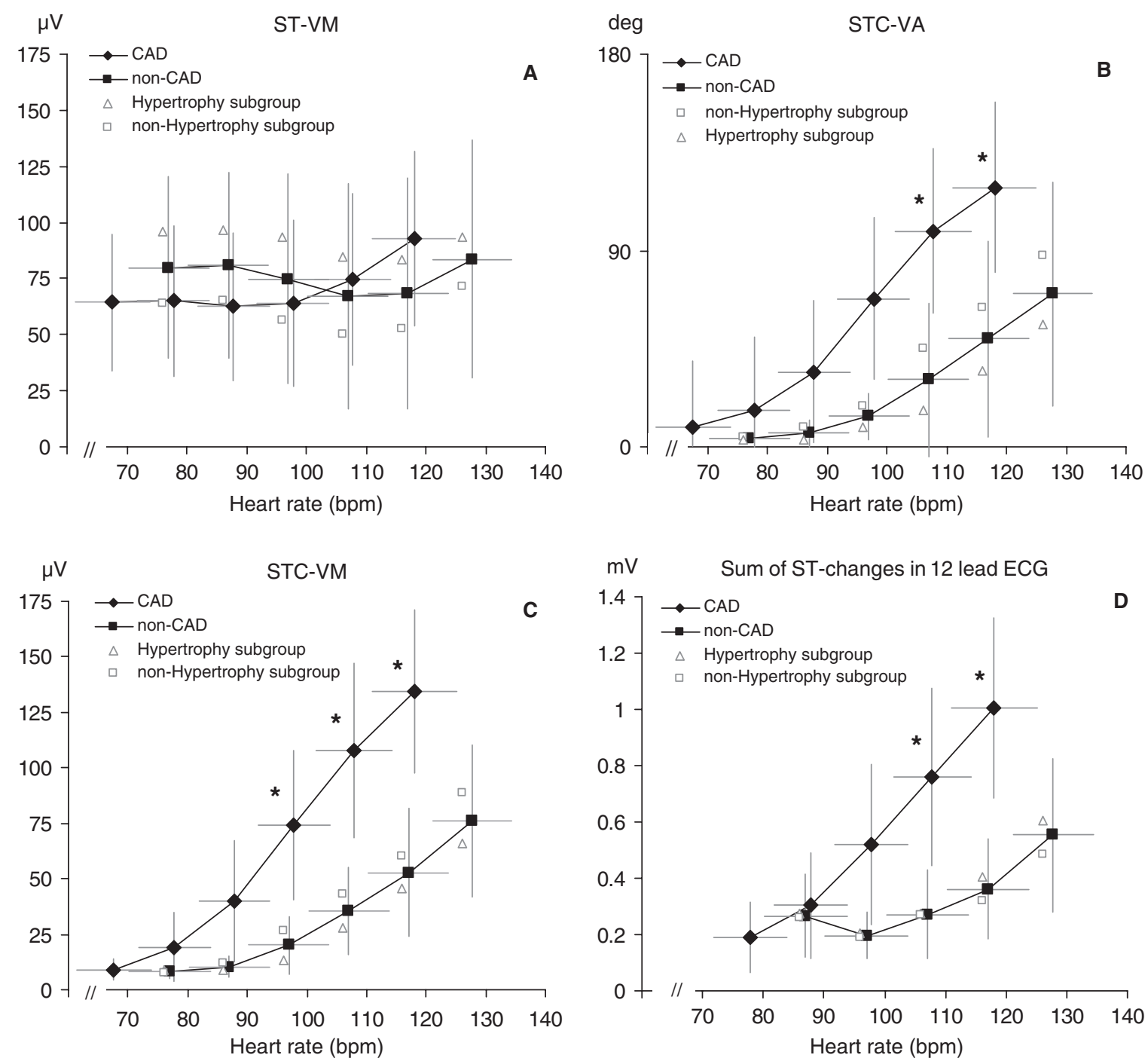

Fig. 2. These panels demonstrate mean vectorcardiography- and electrocardiography-based ST sum values, grouped for heart rates (HR), in the coronary artery disease $(C A D)$ (solid diamonds, $\mathrm{n}=35$ ) and non-CAD patients (solid squares, $\mathrm{n}=10$, open diamonds and open squares, $\mathrm{n}=5)$. STC-VA (B), ST change vector magnitude (STC-VM) (C) and 12-lead ECG ST sum (D) levels all increased in relation to $H R$ during the pacing sequence, and findings for the CAD group were higher at higher HR compared with non-CAD subjects. ST-VM (A) demonstrated no systematic change related to HR for either group, though there was a significant increase in ST-VM for the CAD group at the highest HR. Data are presented as mean \pm standard deviation. ${ }^{*} \mathrm{P}<0.05$ using $\mathrm{t}$-test between groups at each pacing step, with Bonferroni's correction for multiple comparisons.

well as previously reported suggestions for an HR adjustment for the ST level (4) above which STCVM levels should indicate ischemia (see Fig. 4). With these ischemia thresholds, the positive predictive value of STC-VM for ischemia was 0.58 and the negative predictive value was 0.88 .

\section{Discussion}

In this study, we were able to test the relationship of both HR and ischemia to VCG ST results in subjects with established CAD diagnoses, with controlled HR changes and graded 'demand' myocardial ischemia. There appears to be a relatively large component of the total grouped STC-VM values occuring in the CAD group, with a smaller component contributed by HR, as shown in the non-CAD group in Fig. 2C. Although there may be an interaction between HR and ischemia concerning effects on ST levels, there was no way to separate these components in ischemic subjects with this study design, other than to compare them with non-ischemic subjects at the same HR. These results also confirmed previous work concerning ST behavior during $\mathrm{HR}$ increases in 


\section{Contributions of myocardial ischemia and HR to ST segment}

A

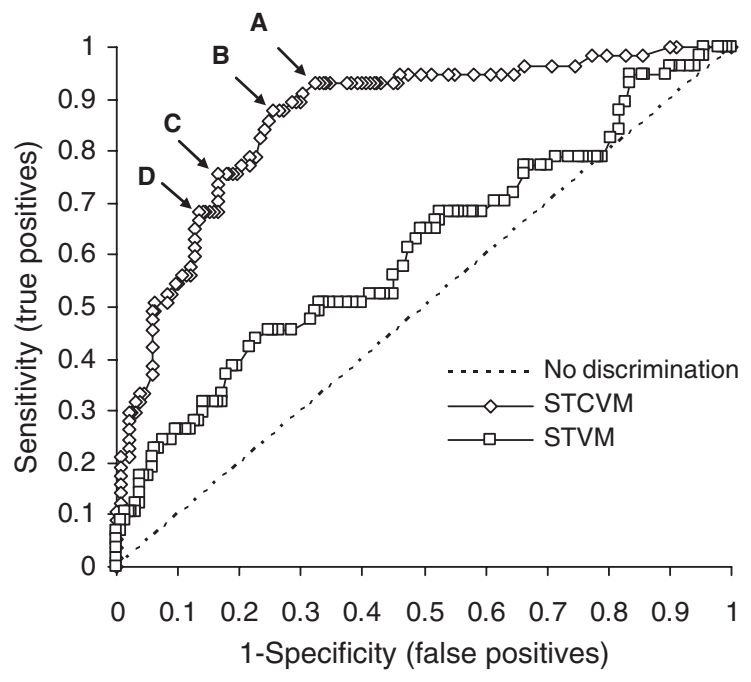

B

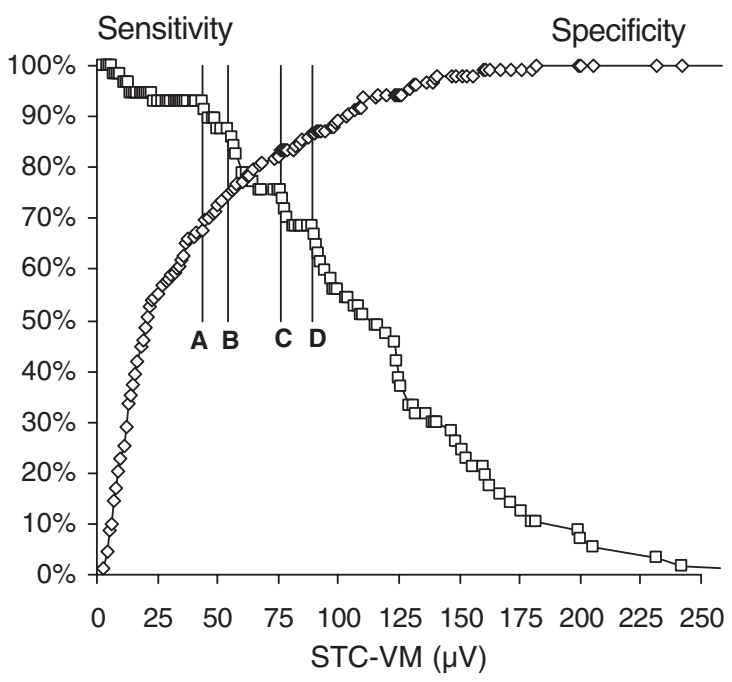

Fig. 3. ROC curves are shown for ST vector magnitude (ST-VM) and STC-VM levels in relation to positive ischemia by lactate analysis (A). ST-VM is little better than chance in this setting for identifying ischemia. In B, sensitivity and specificity for the STC-VM level and positive ischemia are shown, with the same four STC-VM levels identified. It is notable that in the region of these four identified STC-VM levels, sensitivity for identification of ischemia is rapidly declining while specificity rises. This illustrates why a single specific absolute STC-VM threshold has limitations, and that additional factors (such as heart rate) must be included in interpretation of STC-VM levels and likelihood of ischemia.

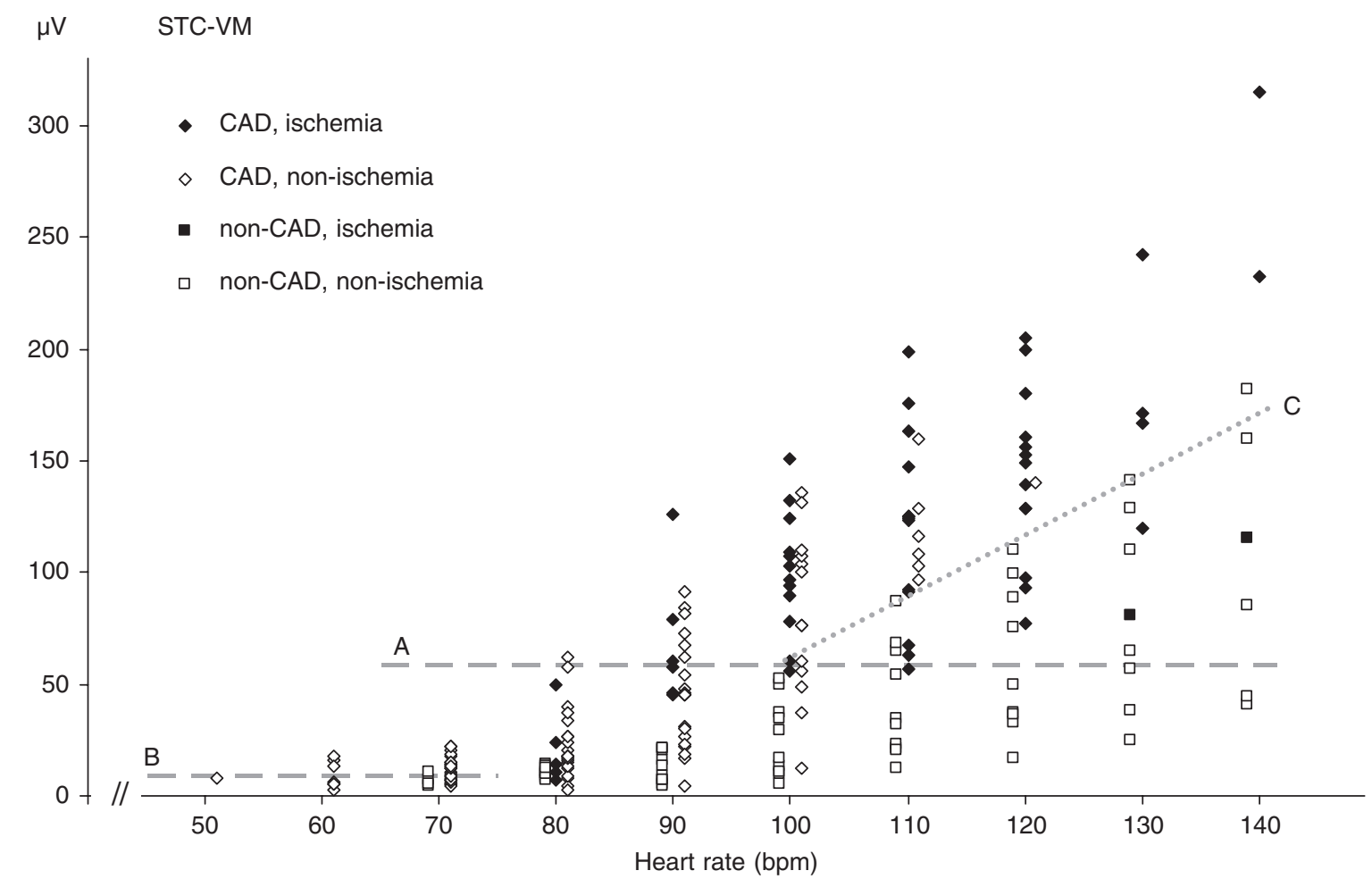

Fig. 4. This figure shows individual measurements, with ischemia and non-ischemia results with ST change vector magnitude (STC-VM) levels marked for both coronary artery disease (CAD) and non-CAD patients. The hatched line labeled ' $A$ ' marks a $\Delta 50 \mu V$ threshold (20, 21), which is placed over the mean 'Baseline' STC-VM value, which is denoted by the hatched line labeled ' $B$ '. The dotted line (C) represents a $2.8 \mu \mathrm{V}$ change per beat (2.0+0.8 standard deviation) for heart rates (HR)s over 100 b.p.m. (4), as a second HR-related STC-VM threshold for ischemia. These thresholds were used to calculate positive and negative predictive values for ischemia based on the STC-VM level. 


\section{S. Häggmark et al.}

non-ischemic conditions $(4,22)$. While the VCG methodology was chosen because of its strengths as a scientific measurement and analysis instrument, these results examine the same ST phenomena reflected by 12-lead ECG in the multiple singlelead planar format, although we have not attempted a comparison of VCG and 12-lead ECG ST analysis. All the 12-lead ECG ST results suggest changes compatible with the VCG findings.

Vector angle change was the main component of STC-VM increase during ischemia as well as HR increases without ischemia. STC-VA during demand ischemia at graded HR levels has not been previously reported. At the same time, ST-VM did not demonstrate a strong relation to ischemia, although changes in ST-VM may have come late in the protocol, at higher degrees of ischemia, and were difficult to characterise systematically. STC$\mathrm{VM}$ changes occurred in the same pattern and magnitude as STC-VA, as can be seen in Fig. 2. STC-VA increased at highest HRs while ST-VM means did not increase significantly, even at highest HRs. Vector angle change has been suggested as an early indicator of ischemia and infarction (23), and can be an additional tool for discrimination between ischemic and postural ECG changes (24, 25). In clinical practice, vector angle change is generally not monitored independently.

These results show that the widely employed and empirically established ischemia thresholds for STVM and STC-VM do not accurately identify ischemia at HRs 100 and above. More dynamic criteria for VCG ST levels are needed, and particularly with regard to higher HR levels. One may implement an ST-HR index or slope factor similar to those proposed for exercise ECG (9, 26-28). An STC-VM threshold for ischemia will have to be a compromise in test performance for sensitivity and specificity, as our findings demonstrate. Specificity of STC-VM for identifying ischemia in this study was unexpectedly low, although this may have been due to limitations in sensitivity of the lactate method as used here to identify the start of ischemia.

To date, no clearly demonstrated VCG ST threshold for myocardial ischemia has been published. Although without reference to $\mathrm{HR}, 50 \mu \mathrm{V}$ (change) has been suggested for ST-VM (20) and STC-VM (29-31). For observations from the angioplasty laboratory, with subjects resting steadily in the supine position, an even lower limit for STC-VM $(25 \mu \mathrm{V})$ has been proposed $(20,29)$. Our material demonstrates a relative contribution of HR to ST magnitude in subjects before and during myocar- dial ischemia, which reflects many clinical settings. While the model that was employed here probably best fits the clinical setting of exercise testing, this is a unique material in that subjects were anesthetised during the ischemic provocation.

The negative predictive value for the ischemic thresholds as tested (STC-VM $50 \mu \mathrm{V}$ increase along with $2.8 \mu \mathrm{V}$ per beat for HR increase above 100 b.p.m.) was high (88\%) while the positive predictive value was lower (58\%). It is likely that the regional lactate production reference method here was not sensitive enough to identify myocardial ischemia rapidly at its onset (where the ECG ST signal might be quicker), and hence there were measurements where ischemia was present and ST elevations occurred, but no lactate production was noted. Similarly, increasingly higher absolute ST levels show rapid declining sensitivity when identifying ischemia. In the ranges of interest in this study, the rate of change in both sensitivity and specificity was rapid for increasing STC-VM levels (Fig. 3B)

Non-CAD subjects increased their myocardial work, as indicated by RPP and metabolism (by $\mathrm{LV} \mathrm{MVO}_{2}$ and lactate consumption), in response to pacing HR steps. The CAD group did not respond the same way. They demonstrated increased LV $\mathrm{MVO}_{2}$ at maximum pacing, though at the same time lactate production. Results for transcoronary lactate flux also corroborate findings of increased myocardial work during pacing, with a simultaneous limited myocardial supply of substrates in the CAD subjects. From this we can summarise that the pacing method produced myocardial demandrelated ischemia in the CAD group, but not in the non-CAD group. Therefore, pacing was an acceptable method for inducing controlled ischemic steps in the CAD subject group.

Some limitations in the study material include the following. The non-CAD group was heterogeneous for both operation-type and myocardial conditions, where half of the subjects had some degree of LV hypertrophy. Also, there were few women in either group. The non-CAD group did not provide enough material from which to generalise about VCG ST behavior during increased HR. There are published reference levels for VCG ST in this setting in awake subjects (4). The numbers in this group were small due to limited access to elective heart operation subjects with minimal ventricular hypertrophy who did not have CAD. The non-CAD group was younger and had a lower incidence of medication ( $\beta$-blocker). Left ventricular mass was presumably greater in some of the non-CAD 
subjects who had valvular heart disease. Left ventricular mass was not measured in all subjects.

Lactate sensitivity and specificity for ischemia is excellent in the laboratory setting, where location and timing of ischemia is known, and lactate sampling is optimal (32). Clinical experience with coronary sinus lactate measurement for detection of ischemia has been described (17-19), and limitations in sensitivity are most likely related to suboptimal sampling of the ischemic myocardial region (33). Lactate measurement as a clinical screening method for myocardial ischemia is inadequate. Still, the specificity of progressive lactate production for myocardial ischemia is very high, which supports our study design of selecting subjects with demonstrated lactate production in order to further analyse the relation of ST-VM and STC-VM to confirmed myocardial ischemia, independent of HR.

In summary, we present a unique clinical material with metabolic measurement for identification of myocardial ischemia during controlled HR increases where the relation of ST changes is tested for both HR and ischemia. We conclude that during assessment of patients for the presence or absence of myocardial ischemia, HR must be taken into account when interpreting VCG ST findings. The diagnostic implication is that in non-hypertrophic patients, an HR adjustment for the ST level may be motivated at higher HRs in order to increase the diagnostic specificity of ST levels for detection of myocardial ischemia. Further prospective validation is needed to establish HR-related VCG ST thresholds for myocardial ischemia.

\section{Acknowledgements}

We wish to acknowledge Per Hohner, MD (now deceased), same department, who was the central figure in the start of this study. The authors gratefully acknowledge the contributions of Margareta Östman and Barbro Österland with assistance for data collection. Sources of financial support include The Swedish Medical Research Council, The Swedish Heart-Lung Fund, The Heart Foundation of Northern Sweden, and Umeå University.

\section{References}

1. Sederholm M, Erhardt L, Sjögren A. Continuous vectorcardiography in acute myocardial infarction. Natural course of ST and QRS-vectors. Int J Cardiol 1983; 4: 53-63.

2. Dellborg M, Topol EJ, Swedberg K. Dynamic QRS complex and ST segment vectorcardiographic monitoring can identify vessel patency in patients with acute myocardial infarction treated with reperfusion therapy. Am Heart J 1991; 122: 943-8.
3. Chou TC. When is the vectorcardiogram superior to the scalar electrocardiogram? J Am Coll Cardiol 1986; 8: 791-9.

4. Häggmark S, Haney MF, Jensen SM et al. ST-segment deviations during pacing-induced increased heart rate in patients without coronary artery disease. Clin Physiol Funct Imaging 2005; 25: 246-52.

5. Okin PM, Kligfield P. Heart rate adjustment of ST segment depression and performance of the exercise electrocardiogram: a critical evaluation. J Am Coll Cardiol 1995; 25 1726-35.

6. Viik J, Lehtinen R, Malmivuo J. Detection of coronary artery disease using maximum value of ST/HR hysteresis over different number of leads. J Electrocardiol 1999; 32 (Suppl.):70-5.

7. Lachterman B, Lehmann KG, Detrano R et al. Comparison of ST segment/heart rate index to standard ST criteria for analysis of exercise electrocardiogram. Circulation 1990; 82: 44-50.

8. Lehtinen R, Sievänen H, Viik J et al. Accurate detection of coronary artery disease by integrated analysis of the STsegment depression/heart rate patterns during the exercise and recovery phases of the exercise electrocardiography test. Am J Cardiol 1996; 78: 1002-6.

9. Kligfield P, Okin PM. Heart rate adjustment of ST depression in patients with coronary disease and negative standard exercise tests. J Electrocardiol 1999; 32 (Suppl.):193-7.

10. Chen L, Ma L, de Prada VA et al. Effects of beta-blockade and atropine on ischemic responses in left ventricular regions subtending coronary stenosis during dobutamine stress echocardiography. J Am Coll Cardiol 1996; 28: 1866-76.

11. Bålfors E, Häggmark S, Ariola Jr S et al. In vitro analysis of thermal transport in coronary sinus thermodilution catheters. Clin Physiol 1983; 3: 469-76.

12. Gwechenberger $M$, Schreiber W, Kittler $\mathrm{H}$ et al. Prediction of early complications in patients with acute myocardial infarction by calculation of the ST score. Ann Emerg Med 1997; 30: 563-70.

13. Frank E. Accurate, clinically practical system for spatial vectorcardiography. Circulation 1956; 13: 737-44.

14. Näslund U, Häggmark S, Johansson G et al. Quantification of myocardium at risk and detection of reperfusion by dynamic vectorcardiographic ST segment monitoring in a pig occlusion-reperfusion model. Cardiovasc Res 1993; 27: 2170-8.

15. Opie LH, Owen P, Thomas M et al. Coronary sinus lactate measurements in assessment of myocardial ischemia. Am J Cardiol 1973; 32: 295-305.

16. Gibbons RJ, Balady GJ, Bricker JT et al. ACC/AHA 2002 guideline update for exercise testing: summary article: a report of the American College of Cardiology/American Heart Association Task Force on practice guidelines (committee to update the 1997 exercise testing guidelines). Circulation 2002; 106: 1883-92.

17. Cohen LS, Elliott WC, Klein MD et al. Coronary heart disease. Clinical, cinearteriographic and metabolic correlations. Am J Cardiol 1966; 17: 153-68.

18. Parker JO, Chiong MA, West RO et al. Sequential alterations in myocardial lactate metaboli, S-T segments, and left ventricular function during angina induced by atrial pacing. Circulation 1969; 40: 113-31.

19. Gertz EW, Wisneski JA, Neese R et al. Myocardial lactate metabolism: evidence of lactate release during net chemical extraction in man. Circulation 1981; 63: 1273-9.

20. Dellborg M, Emanuelsson $H$, Riha $M$ et al. Dynamic QRS complex and ST segment monitoring by continuos vectorcardiography during coronary angioplasty. Coronary Artery Dis 1991; 2: 43-52. 


\section{S. Häggmark et al.}

21. Jensen SM, Häggmark S, Johansson G et al. On-line computerized vectorcardiography: influence of body position, heart rate, radiographic contrast fluid and myocardial ischemia. Cardiology 1997; 88: 576-84.

22. Häggmark S, Haney MF, Johansson $G$ et al. Vectorcardiographic ST deviations related to increased heart rate in the absence of ischemia in an experimental pig model. $J$ Electrocardiol 2006; 39: 169-76.

23. Näslund U, Häggmark $S$, Johansson G et al. Ischaemia and reperfusion induced transient QRS vector changes: relationship to size of the ischaemic territory. Cardiovasc Res 1993; 27: 327-33.

24. Schmitt C, Lehmann G, Schmieder S et al. Diagnosis of acute myocardial infarction in angiographically documented occluded infarct vessel: limitations of ST-segment elevation in standard and extended ECG leads. Chest 2001; 120: $1540-6$.

25. Pharand C, Nasmith JB, Rajaonah JC et al. Distinction between myocardial ischemia and postural changes in continuous ECG monitoring based on ST-segment amplitude and vector orientation-preliminary results. Can J Cardiol 2003; 19: 1023-9.

26. Okin PM, Anderson KM, Levy D et al. Heart rate adjustment of exercise-induced ST segment depression. Improved risk stratification in the Framingham offspring study. Circulation 1991; 83: 866-74.

27. Morise AP. Accuracy of heart rate-adjusted ST segments in populations with and without posttest referral bias. Am Heart J 1997; 134: 647-55.

28. Bjurö T, Gullestad L, Endresen K et al. Evaluation of STsegment changes during and after maximal exercise tests in one-, two- and three-vessel coronary artery disease. Scand Cardiovasc J 2004; 38: 270-7.

29. Jensen SM, Johansson G, Osterman G et al. On-line computerized vectorcardiography monitoring of myocardial ischemia during coronary angioplasty: comparison with 12-lead electrocardiography. Coron Art Dis 1994; 5: 507-14.

30. Lundin P, Eriksson SV, Fredrikson M et al. Prognostic information from on-line vectorcardiography in unstable angina pectoris. Cardiology 1995; 86: 60-6.

31. Jensen SM, Karp K, Rask P et al. Assessment of myocardium at risk with computerized vectorcardiography and technetium-99m-sestamibi-single photon emission computed tomography during coronary angioplasty. Scand Cardiovasc J 2002; 36: 11-8.

32. Wikström G, Ronquist G, Nilsson $S$ et al. Continuous monitoring of energy metabolites using microdialysis during myocardial ischaemia in the pig. Eur Heart J 1995; 16: 339-47.

33. Crittenden MD. Intraoperative metabolic monitoring of the heart: I. Clinical assessment of coronary sinus metabolites. Ann Thorac Surg 2001; 72: S2220-6 discussion S22.

Address:

Sören Häggmark

Heart Centre

University Hospital of Umeå

SE-901 85 Umeå

Sweden

e-mail: soren.haggmark@vll.se 